\title{
APROBACIÓN DEL PLAN REGULADOR COMUNAL DE NUUÑOA A TRAVÉS DEL USO CREATIVO DE LA NORMATIVA*
}

Approving the zoning plan of Nuñoa through the creative use of the rules

\section{MATTHEW WELLINGTON CAULKINS**}

RESUMEN: A propósito de la incertidumbre jurídica que un decreto reciente del Ministerio de Vivienda y Urbanismo (MINVU) trata de solucionar, el presente artículo analiza la aplicación de un instrumento normativo en un caso de planificación urbana local. Se propone que esa aplicación se entiende mejor con un enfoque en el uso estratégico de la normativa. Los instrumentos normativos no se aplican de forma mecánica sino de forma selectiva y con orientación a cumplir objetivos de forma creativa. Se revisa un estudio de caso de forma interpretativa y basada en entrevistas y revisión de documentos, es el caso conflictivo de la aprobación de una modificación integral al Plan Regulador Comunal de la municipalidad de Nuñoa frente a una significativa insatisfacción ciudadana. El análisis demuestra la importancia de la representación de la planificación como la mera aplicación de sus instrumentos normativos y la potencia de un enfoque en la aplicación de los instrumentos normativos para entender la gobernanza, demostrando el cumplimiento selectivo y creativo de la Ordenanza General de Urbanismo y Construcción. Se insiste que cualquier movilización por crear instrumentos normativos más justos debe ser complementada por un esfuerzo de analizar la aplicación de esos instrumentos.

PALABRAS CLAVES: Instrumentos de planificación urbana, cumplimiento creativo, uso de la normativa.

ABSTRACT: Regarding the legal uncertainty that a recent decree of the Ministry of Housing and Urban Planning tries to resolve, this article analyzes the application of planning instruments in a case within Chilean urban planning institutions. The argument proposed here is that application of planning tools is best understood through the lens of the strategic use of rules. Planning instruments are not applied mechanically but selectively and with an orientation to the creative achievement of goals. The paper presents an interpretive case study based on interviews and documentary analysis. A conflictive case is reviewed, the approval of a modification to a zoning plan in the municipality of Nuñoa in the face of significant citizen dissatisfaction. The analysis demonstrates the importance of the representation of planning as mere application of normative instruments and the usefulness of a focus on the application of planning instruments to understand governance and demonstrate selective and creative compliance of regulations. The article insists any mobilization to create more just planning tools must be complemented by an effort to analyze their application as well.

KEY WORDS: Planning tools, creative compliance, use of the rules.

Recibido: 23.06.2020 Aceptado: 09.07.2020

* El autor agradece el apoyo de Alberto Gurovich con la investigación inicial y de Ruth Beilin y Mauro Fontana con el desarrollo conceptual del análisis. 


\section{INTRODUCCIÓN}

Recientemente la División de Desarrollo Urbano del Ministerio de Vivienda y Urbanismo len adelante MINVU) emitió un decreto buscando dar mayor seguridad al mercado nacional sobre la aplicación del marco normativo que rige a la construcción. Sin embargo, el decreto no hace más que afirmar hechos evidentes cuando dice que los "permisos, aprobaciones y autorizaciones emitidas por los Directores de Obras Municipales gozan de una presunción de legalidad, de imperio y exigibilidad frente a sus destinatarios" (Decreto 14, 2019). No tardó más de dos días para que titulares insistieran que el decreto no lograría resolver la "incertidumbre jurídica" (Gotschlich y Miranda, 2019). El presente artículo parte de la comprensión reduccionista de la aplicación de los instrumentos de la planificación en la literatura especializada de planificación urbana para proponer como hipótesis que la aplicación de los instrumentos normativos es intencional y creativa, lo cual se revisa a través de un caso conflictivo de la planificación urbana local.

El estudio se enfoca en la importancia de las acciones dentro de los diversos niveles institucionales de la planificación. Contrario al ideal de que las decisiones se limitan a la esfera política y su aplicación sin discreción a la esfera burocrática (Svara, 2001), las acciones en la planificación local son acciones orientadas a objetivos, creativos y selectivos en su utilización de distintos elementos de la normativa. La normativa conforma lo que se denomina un campo normativo, un espacio complejo donde distintos cuerpos normativos se sobreponen y el cumplimiento de cualquier norma específica depende de una complicada evaluación táctica por parte de los actores institucionales respecto a la necesidad y la utilidad de aplicar a esa norma en particular, siendo este argumento una pieza clave para entender que cualquier movilización social por crear normativas más justas debe incluir un enfoque hacia su aplicación.
Para entender ese espacio de incertidumbre en la aplicación de la normativa se presenta un caso asociado a la construcción de la edificación en altura y la participación de un grupo de acción ciudadana local en la aprobación del Plan Regulador Comunal de Ñuñoa entre 2004 y 2007. En ese momento, se buscaba aumentar la densidad del área municipal en cumplimiento al Plan Intercomunal de Santiago de 1994 y frente a una oposición ciudadana potente. Debido a un aumento notoria de la altura de edificación entre 2004 y 2006 (ver figura 1) distintos grupos ciudadanos se organizaron, dando a conocer su descontento en los medios de comunicación locales y nacionales. Colectaron firmas en las plazas locales exigiendo una moratoria respecto a la aprobación de edificación en altura sobre los cuatro pisos y una discusión ciudadana amplia del plan (Aguirre y León, 2007). Aunque el caso habla sobre un Plan Regulador Comunal, el enfoque del estudio no está ahí sino en la aplicación creativa y selectiva de otro instrumento normativo la Ordenanza General de Urbanismo y Construcciones (OGUC) - necesario para aprobar al Plan Regulador Comunal nuevo.

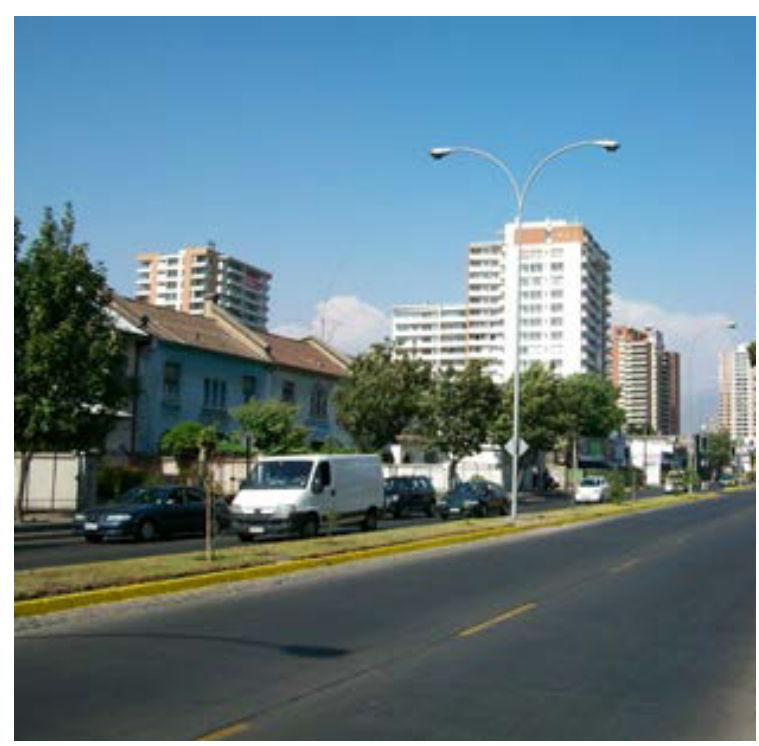

Figura 1. Aumento de la edificación en altura en la avenida Irarrázaval (Zona 1). Fuente: Elaboración propia. 


\section{MARCO TEÓRICO Instrumentos normativos y su aplicación}

Varios son los autores que insisten en la importancia de un análisis que empiece a abrir a la "caja negra" de la implementación en la planificación (Hupe y Hill, 2016; Loh, 2017). Sin embargo, en las investigaciones en el campo de la planificación urbana hay una tendencia en confundir la existencia de instrumentos normativos con su implementación. Algunos estudios analizan cómo actores políticos o técnicos han realizado ajustes a los instrumentos de planificación para lograr sus objetivos. En este sentido, Vicuña (2013) explica cómo se han hecho ajustes a los instrumentos de planificación urbana buscando beneficiar a los empresarios de la construcción. Otros estudios identifican distintas deficiencias de la planificación local respecto a incluir mejor a la participación ciudadana (Herrmann y Van Klaveren, 2016), reducir el sprawl de las ciudades (Gomez y Moris, 2017) o tomar en cuenta factores de riesgo (Villagra, Herrmann, Quintana y Sepúlveda, 2016). En cada caso, se propone modificar a los instrumentos de planificación urbana existentes para incorporar a la materia estudiada, entendiendo así, que la implementación vendría incorporada en la existencia de los nuevos instrumentos propuestos, olvidando el aprendizaje social necesario para que la aprobación de nuevos instrumentos llegue a afectar a las acciones de amplios sectores de la sociedad. Así, se aprecia que en la literatura enfocada en la planificación urbana no se discute cómo se aplican los instrumentos normativos. Debido a esa omisión se perpetúa la idea de que los instrumentos se aplican de forma mecánica.

Por otro lado, desde las investigaciones sobre la administración pública surge la hipótesis de que los instrumentos normativos se aplican de forma intencional y creativa (Batory, 2016; Wagenaar, 2014). En ese sentido, hay varios estudios donde podemos percibir como esa implementación ocurre en un mundo práctico que se caracteriza por su orientación a la acción. Esas acciones, aunque gatilladas por reglas, no necesariamente las obedecen de forma sencilla cuando, por ejemplo, funcionarios europeos de la inmigración deciden si ciertas normas pueden ser creíblemente evitadas para favorecer un cliente o si evitarlas les costaría una reprimenda de sus superiores (Wagenaar, 2004). Una trabajadora social holandesa decide de forma intencional cuando limita los beneficios entregados a las personas sin techo según reglamentos y cuando busca una manera creativa de garantizar la salud y seguridad de sus clientes, aun a pesar de esos reglamentos (Wagenaar, 2006).

En la misma línea, pero en materia de planificación urbana, Throgmorton (2003) explica cómo los urbanistas crean "historias" a través de la caracterización de personajes, escenarios, ritmos e imágenes con los documentos oficiales y minutas que producen en su labor cotidiana. Estas historias guían a la atención de los participantes en los conflictos en esta dirección y no aquella. También Batory (2016), explica cómo los burócratas cumplen normativas de forma creativa, distinguiendo el cumplimiento (meramente) simbólico que busca aparentar cumplir una regla nueva sin la necesidad de cambiar rutinas establecidas.

Al perseguir sus objetivos, los actores institucionales buscan "lo que funciona" en diferentes situaciones. Sus acciones siempre son intencionales, ya que cada actor busca alcanzar sus propios objetivos. Sin embargo, la acción siempre tiene lugar en el "presente eternamente en desarrollo" (Cook y Wagenaar, 2012, traducción del autor), donde los actores tienen una comprensión limitada de las acciones de todos los otros actores. Volviendo al ámbito de la planificación urbana local, un interesante estudio de Chia, Vitry y Negrete (2016) revisa cómo los instrumentos normativos sirven para coordinar a la acción en un territorio. Su implementación no ocurre por la sola existencia de esos instrumentos, 
de la interacción de los variados actores sociales e institucionales y de su aprendizaje colectivo respecto a la gobernanza. Esto es, tienen que generarse nuevos conocimientos y nuevas prácticas para que nuevos instrumentos lleguen a apropiarse.

La implementación de las normativas burocráticas es en sí misma una acción estratégica compleja más que una acción sin reflexión. Heidelberg (2016), a partir del estudio de un caso de fracaso en la aprobación de una política pública en Estados Unidos de América, explica la importancia del enfoque en el ejercicio del poder. No todo fracaso es lo que parece ser. Las acciones administrativas dependen del entendimiento profundo de las normativas por parte de los administradores públicos. Demuestra la importante relación entre los aspectos visibles de las normativas legibles y la dimensión, muchas veces invisible, del uso de esas reglas.

En resumidas cuentas, siempre es necesario que los actores en las instituciones públicas ejerciten discreción al decidir sobre lo útil y lo apropiado de las distintas formas posibles de aplicar una dada normativa. La acción institucional, como toda acción humana, está orientada a objetivos, es creativa y selectiva en la aplicación de las normativas. Los objetivos de los distintos actores pueden ser contrarios a la intención de las normativas, pero el uso de ellas es en gran medida invisible, mientras las normativas mismas quedan más a la vista. A través de una comunicación estratégica, creando "historias" en las palabras de Throgmorton (2003), las acciones en el espacio institucional pueden cumplir de forma simbólica a esas mismas normativas.

\section{METODOLOGÍA}

Este artículo es el resultado de un análisis retrospectivo de una investigación anterior realizada por el autor. El trabajo de estudio de caso descriptivo se realizó entre 2006 y 2007, al momento en que el conflicto en Nuñoa se desarrollaba (Aguirre y León, 2007). Se realizaron entrevistas a actores elegidos por su participación directa en el conflicto por la aprobación de la modificación del Plan Regulador Comunal. Se buscó incorporar actores que aparecían en las discusiones sobre el caso en los medios de comunicación, incluyendo algunos que apoyaban y otros que se oponían a la aprobación del plan. Se entrevistó a tres actores por su participación en la aprobación de la modificación al Plan Regulador Comunal: el director de obras municipales de Nuñoa, la asesora urbanista de la misma municipalidad y el director de la Secretaria Regional Ministerial del Ministerio de Vivienda y Urbanismo len adelante Seremi-MINVU). Entre aquellos que se opusieron a la modificación se entrevistó a una concejala de la municipalidad de Nuñoa y a la directora de un grupo ciudadano llamado Red Ciudadano por Ñuñoa. Las entrevistas se transcribieron en ese entonces. Para el análisis actual, las entrevistas se analizaron según términos claves como aplicación, instrumentos normativos, intención y creatividad (Wagenaar, 2014), para entender cómo la Ordenanza General de Urbanismo y Construcciones se aplicó en el caso. Para comprender el contexto de la aplicación de ese instrumento normativo se revisó el Plan Regulador Comunal propuesto y el existente, las estadísticas de aprobación de permisos de edificación municipales y metropolitanos, se aplicó una encuesta a residentes de los edificios nuevos y se hicieron recorridos por el sector.

\section{RESULTADOS}

El Censo de 2002 había revelado una reducción de la población en la comuna de Nuñoa, de 172.575 en 1992 a 163.511 (ver figura 2), y la densidad había bajado de 102 a menos de 97 hab/ha. Pero en el mismo período el Plan Intercomunal de 
Santiago de 1994 había pedido un aumento de la densidad comunal a 150 hab/ha.

Así, en 2004 la administración municipal de Ñuñoa promulgó un Plan Seccional que aumentaba las facilidades para la construcción en altura en la Zona 1, cercana a la avenida Irarrázaval. El resultado incluyó el aumento de la construcción de edificios en altura, pero creaba trastornos para los vecinos, con incremento de ruido, polvo y camiones en las calles locales, provocando que varios residentes se organizasen en agrupaciones, como la Red Ciudadana por Nuñoa, que tienen su origen en un conflicto entre un edificio en altura y la casa vecina.

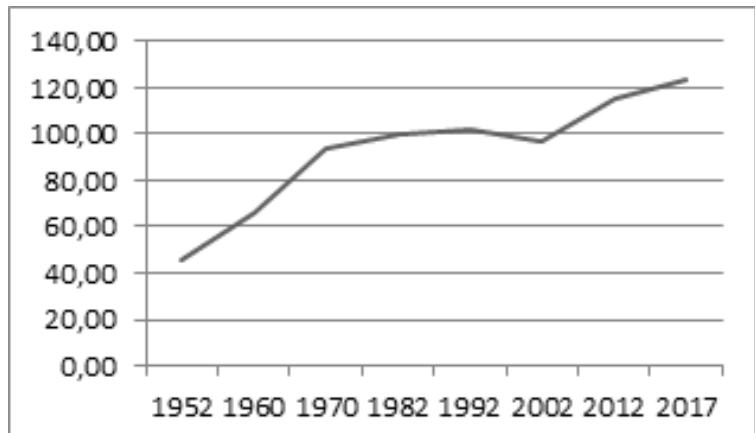

Figura 2. Densidad de la comuna de Ñuñoa (hab/ha). Fuentes: Honold, Correa \& Prieto (1989) y archivos INE (www.ine.cl).

El equipo de planificación municipal, liderado por el alcalde, creó una estrategia para promover la continua densificación de la comuna y neutralizar su oposición a través de una estrategia de tres componentes. Primero, ofrecieron un plan nuevo que era una modificación integral del Plan Regulador Comunal existente, con una reducción de la altura de edificación en las Zonas 3 y 4, aumento en la Zona 2, pero manteniéndola fija en la Zona 1 (ver figura 3). Segundo, ofrecieron una audiencia pública y, tercero, una moratoria que congelaba la altura de edificación en la comuna, implicando que no se podían aprobar permisos por sobre la nueva normativa que aún estaba en proceso de aprobación. Esta postergación de otorgamiento de permisos, dictado por resolución de Seremi-MINVU, tiene la validez máxima de un año y no se podría extender (OGUC, artículo 1.4.18). De esa forma, el plan parecía entregar todo lo que las agrupaciones ciudadanas exigían, ofreciendo una reducción de las alturas de edificación en dos de las cuatro zonas de la comuna, una posibilidad de discusión del plan (una audiencia pública) y, mientras el plan todavía no se aprobaba, ofrecía una moratoria que instalaba esa altura más baja desde ese mismo momento. Junto a eso se incluía un aumento de altura en un sector (Zona 2) y se mantenía la altura aumentada recientemente en otro (en la Zona 1). El Director de Obras Municipal explicó que, con esas dos concesiones, se buscaba "alejar" las edificaciones en altura "hacia los bordes" de la comuna (hacia la Zona 2) para así despejar el centro.

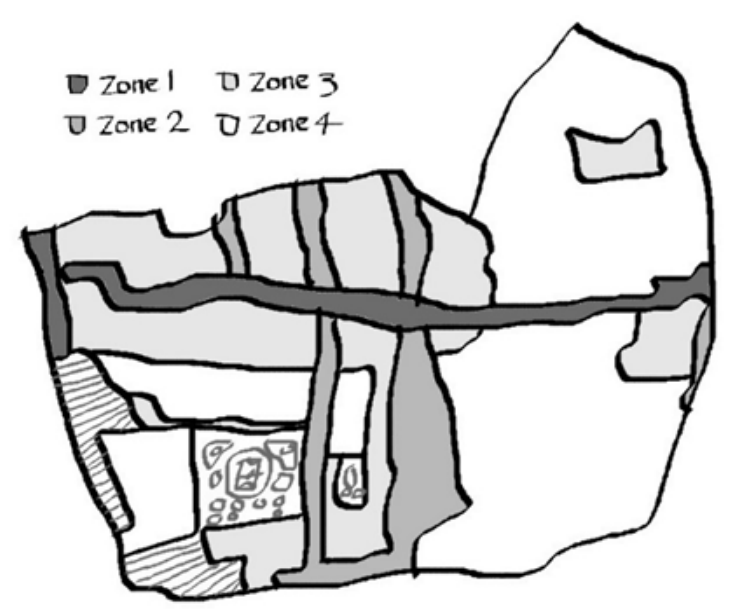

Figura 3. Zonas del Plan Regulador Comunal de Nuñoa. Fuente: Elaboración propia a partir de Plan Regulador Comunal de Ñuñoa.

Lo que inicialmente parecía un plan conciliatorio, escondía acciones más estratégicas. El equipo de planificación usó su experiencia acumulada de décadas en el uso de las normativas para aparentar tres concesiones, mientras en realidad lograban algunas pequeñas ganancias en altura, la mantención de las ganancias del Plan Seccional de 2004 y cimentar la aprobación de su plan.

Primero, la reducción de altura en las Zonas 3 y 4 era sólo simbólica. En la Zona 3, 
efectivamente se rebajaba la altura, de altura libre a 5 y 7 pisos (dependiendo del tamaño del terreno). Pero, de acuerdo con entrevista con una concejala, esa Zona 3 ya estaba prácticamente "saturada" de edificios en altura, de manera que la concesión era menos generosa de lo que parecía ser.

Pero aún menos generosa fue la reducción en la Zona 4, debido a que esa zona antes era de 4 pisos y la propuesta era bajarla a 3 pisos. Sin embargo, leyendo con cuidado la normativa propuesta se descubría otra intención, la cual se relacionaba con la reducción que otorgaba sólo en terrenos de menos de mil metros cuadrados, pero en aquellos de más de mil metros cuadrados existía un aumento de la altura que pasaba de 4 a 5 pisos.

Segundo, la audiencia pública es obligatoria para todo proceso de aprobación o modificación de Plan Regulador Comunal. Según la directora de la Red Ciudadana por Nuñoa, presentarlo como una concesión hacia la oposición sirvió para representar al equipo de planificación municipal en los medios con una actitud y comportamiento generoso hacia una oposición obstinada, sirviendo de esa manera para neutralizar el apoyo de los grupos ciudadanos en los medios de comunicación. Además, la audiencia pública dictada por la Ordenanza no es un momento para discusión de un plan, sino que de información para la población local respecto éste (OGUC, artículo 2.1.111), a través del cual los residentes pueden hacer llegar cualquier crítica por carta a la municipalidad, pero que, en la práctica, tampoco es participativa.

Tercero, la moratoria sirvió para poner en efecto la nueva normativa antes de aprobado el plan, demostrando creatividad por parte del equipo de planificación local, debido a que seleccionaron la norma de acuerdo con sus propios objetivos. La moratoria sirvió para influenciar a los concejales para que no existiera ninguna discusión efectiva del plan.

El alcalde utilizó su experiencia con la normativa que rige el proceso de aprobación de planes reguladores para presionar a los concejales a aceptar la propuesta tal como fue originalmente concebida. Esa presión significó que no se pudo aceptar ninguna de las críticas expresadas por los vecinos, aunque muchas cartas habían sido recibidas. Según la concejala entrevistada, el alcalde insistía que no se podía aceptar ninguna sugerencia que hiciera una modificación a la propuesta, porque hubiese implicado una nueva audiencia con un nuevo plazo para el envío de cartas, alargando el proceso mucho más allá del año.

En resumen, el equipo utilizó su experiencia con la gran variedad de normas aplicables, debido a que conocían en profundidad la normativa de los Planes Reguladores Comunales y de su proceso de aprobación en la Ordenanza General de Urbanismo y Construcciones, a tal punto, de representar simbólicamente un aumento de altura en la Zona 4 como una reducción y desarrollar una audiencia pública como una concesión de un espacio participativo. Además, utilizaron de forma estratégica la norma poco conocida de la postergación de otorgamiento de permisos de forma muy perspicaz para poner presión al proceso, no dejando ni a los concejales ni a los vecinos modificar a la propuesta. Al final del proceso, según entrevista con el entonces director de la Seremi-MINVU, utilizaron sus contactos en esa institución para acelerar la aprobación de la modificación dentro de lo que quedaba del acotado plazo de un año.

\section{DISCUSIÓN Y CONCLUSIONES}

En este artículo se buscó comprender la incertidumbre que aqueja actualmente al Ministerio de Vivienda y Urbanismo y los Directores de Obras Municipales. Para eso se revisó la literatura respecto a la planificación urbana que presenta los instrumentos normativos como único elemento visible y deja de lado el uso que se hace de esa normativa, como si su aplicación, por parte de los actores institucionales y sociales, fuera mecánica. La literatura revisada sobre administración pública 
propuso la hipótesis de que las acciones que se desarrollan en las instituciones públicas son creativas, selectivas y orientadas a objetivos.

Se revisó el caso de estudio de la aprobación de la modificación integral al Plan Regulador Comunal de Ñuñoa en 2007 y se analizó cómo las acciones del equipo de planificación municipal ejercieron discreción, creatividad y cumplimiento simbólico de la normativa de la Ordenanza General de Urbanismo y Construcción que se refiere a las aprobaciones deplanesnuevososus modificacionesintegrales, reconociendo tres componentes del proceso como concesiones a la oposición, amarrando así su apoyo al plan. Sin embargo, un análisis más detenido demuestra que los tres elementos fueron sólo representados como concesiones, siendo meramente concesiones simbólicas.

El análisis del caso demuestra que los instrumentos de planificación urbana no se aplican de forma mecánica, sino que su aplicación depende de los objetivos de los actores institucionales que actúan con intención y creatividad. Esa discusión cobra más importancia en la actual contingencia, porque implica que cualquier movilización social por crear instrumentos normativos más justos debe también incluir un esfuerzo por la aplicación adecuada de esas normativas.

\section{REFERENCIAS BIBLIOGRÁFICAS}

Aguirre, C. \& León, D. (2007) Análisis descriptivo de la evolución urbana de la comuna de Nuñoa 2001 a 2006. Revista Urbano, (16), 60-72. Recuperado de http://revistas. ubiobio.cl/index.php/RU/article/view/377

Batory, A. (2016). Defying the Commission: Creative Compliance and Respect for the Rule of Law in the EU. Public Administration, 94(3), 685-699. https:// doi.org/10.1111/padm. 12254

Chia, E., Vitry, C., \& Negrete, J. (2016). La producción de sentido y contextualización de los instrumentos de planificación territorial. Los casos de Lunelois (Francia) y Quilpué (Chile). Revista geográfica de Valparaíso, (53), 47 71. Recuperado de http://revistageografica. cl/index.php/revgeo/article/view/4/4

Cook, S. N., \& Wagenaar, H. (2012). Navigating the eternally unfolding present: Toward an epistemology of practice. The American Review of Public Administration, 42(1), 3-38. https://doi. org/ 10.1177\%2F0275074011407404

Decreto 14. Diario Oficial de la República de Chile, Santiago, Chile, 30 de septiembre de 2019.

Gotschlich, D. \& Miranda, P. (2019). Expertos advierten que decreto de Vivienda no resuelve la "incertidumbre jurídica". El Mercurio, Nacional, p. Cl.

Gomez, T., \& Moris, R. (2017). Ciudades Solares: Una Mirada desde la Planificacion Urbana. Revista Planeo. Ciudades Inclusivas, (62), 1-15. Recuperado de http: / / revistaplaneo.cl/wp-content/ uploads/Art\%C3\%ADculo_GomezMoris.pdf

Heidelberg, R. (2016). The power of knowing the rules. The American Review of Public Administration, 46(6), 734-750. https://doi. org/10.1177\%2FO275074015575353

Herrmann, M. G., \& Van Klaveren, A. (2016). Disminución de la participación de la población en organizaciones sociales durante los últimos trece años en Chile e implicaciones para la construcción de una política de planificación urbana más participativa. EURE (Santiago), 42(125), 175-203. http://dx.doi.org/10.4067/ SO250-71612016000100008

Honold, J., Correa, P. \& Prieto, M. (1989). Memoria Explicativa - Plan Regulador Comunal de Ñuñoa. Santiago, Chile: llustre Municipalidad de Ñuñoa.

Hupe, P. L. \& Hill, M. J. (2016). 'And the rest is implementation.' Comparing approaches 
to what happens in policy processes beyond Great Expectations. Public Policy and Administration, 37(2), 103-121. https://doi. org/10.1177\%2F0952076715598828

Svara, J. (2001). The Myth of the Dichotomy: Complementarity of Politics and Administration in the Past and Future of Public Administration. Public Administration Review, 61(2), 176-183. https://www.jstor.org/ stable/97745 1 ? seq = 1

Throgmorton, J. A. (2003). Planning as persuasive storytelling in a global-scale web of relationships. Planning Theory, 2(2), 125-151. https://doi. org/10.1177\%2F14730952030022003

Vicuña del Río, M. (2013). El marco regulatorio en el contexto de la gestión empresarialista y la mercantilización del desarrollo urbano del Gran Santiago, Chile. Revista INVI, 28(78), 181-219. http://dx.doi.org/10.4067/ s0718-83582013000200006

Villagra, P., Herrmann, G., Quintana, C., \&
Sepúlveda, R. D. (2016). El pensamiento resiliente y la planificación urbana en un entorno costero bajo riesgo de tsunami: el caso de Mehuín, Chile. Revista de Geografía Norte Grande, (64), 55-62. http://dx.doi.org/10.4067/S071834022016000200005

Wagenaar, H. (2014). Meaning in action: Interpretation and dialogue in policy analysis. Abingdon: Routledge.

Wagenaar, H. (2006). Bureaucratic order and personal order: the narrative analysis of administrative practice. En M. A. van den Brink \& T. Metze (Eds.), Words matter in policy and planning. Discourse theory and method in the social sciences (pp. 43-63). Utrecht: Nederlandse Geografische Studies.

Wagenaar, H. (2004). 'Knowing' the rules: administrative work as practice. Public Administration Review, 64(6), 643656. https://doi.org/10.1111/i.1540$6210.2004 .00412 . x$ 